Knežević,Krčadinac,Relić Matrix products of binomial coefficients ...

\title{
MATRIX PRODUCTS OF BINOMIAL COEFFICIENTS AND UNSIGNED STIRLING NUMBERS
}

\author{
MARIN KNEŽEVIĆ, VEDRAN KRČADINAC, AND LUCIJA RELIĆ \\ Dedicated to Professor Dragutin Svrtan on the occasion of his $70^{\text {th }}$ birthday.
}

\begin{abstract}
We study sums of the form $\sum_{k=m}^{n} a_{n k} b_{k m}$, where $a_{n k}$ and $b_{k m}$ are binomial coefficients or unsigned Stirling numbers. In a few cases they can be written in closed form. Failing that, the sums still share many common features: combinatorial interpretations, Pascal-like recurrences, inverse relations with their signed versions, and interpretations as coefficients of change between polynomial bases.
\end{abstract}

MSC: 05A10

\section{INTRODUCTION}

Let $a_{n k}$ and $b_{n k}$ be binomial coefficients $\left(\begin{array}{l}n \\ k\end{array}\right)$ or unsigned Stirling numbers of the first and second kind: $\left[\begin{array}{l}n \\ k\end{array}\right]$ and $\left\{\begin{array}{l}n \\ k\end{array}\right\}$ in Karamata-Knuth notation, respectively. We are interested in the sum $\sum_{k=m}^{n} a_{n k} b_{k m}$. Denote by $A=\left[a_{n k}\right]$ and $B=\left[b_{n k}\right]$ the corresponding infinite lowertriangular matrices, indexed by the non-negative integers. The sum can be interpreted as the $(n, m)$-entry of the matrix product $A \cdot B$.

Our motivation are the following two sums, that can be written in closed form:

$$
\begin{aligned}
\sum_{k=m}^{n}\left(\begin{array}{l}
n \\
k
\end{array}\right)\left\{\begin{array}{l}
k \\
m
\end{array}\right\} & =\left\{\begin{array}{c}
n+1 \\
m+1
\end{array}\right\}, \\
\sum_{k=m}^{n}\left[\begin{array}{l}
n \\
k
\end{array}\right]\left(\begin{array}{l}
k \\
m
\end{array}\right) & =\left[\begin{array}{c}
n+1 \\
m+1
\end{array}\right] .
\end{aligned}
$$

These are identities (6.15) and (6.16) in the book Concrete Mathematics [1]. Furthermore, the following sum are the Lah numbers [2, 3], denoted by $L(n, m)$ :

$$
\sum_{k=m}^{n}\left[\begin{array}{l}
n \\
k
\end{array}\right]\left\{\begin{array}{c}
k \\
m
\end{array}\right\}=\frac{n !}{m !}\left(\begin{array}{c}
n-1 \\
m-1
\end{array}\right)
$$

DOI: https://doi.org/10.5592/CO/CCD.2020.04 
Knežević,Krčadinac,Relić Matrix products of binomial coefficients ...

\begin{tabular}{|cc|cc|cc|}
\hline$\sum_{k}\left(\begin{array}{l}n \\
k\end{array}\right)\left(\begin{array}{l}k \\
m\end{array}\right)$ & $\mathrm{A} 038207$ & $\sum_{k}\left(\begin{array}{l}n \\
k\end{array}\right)\left[\begin{array}{c}k \\
m\end{array}\right]$ & $\mathrm{A} 094816$ & $\sum_{k}\left(\begin{array}{l}n \\
k\end{array}\right)\left\{\begin{array}{c}k \\
m\end{array}\right\}$ & $\mathrm{A} 008277$ \\
\hline$\sum_{k}\left[\begin{array}{l}n \\
k\end{array}\right]\left(\begin{array}{c}k \\
m\end{array}\right)$ & $\mathrm{A} 130534$ & $\sum_{k}\left[\begin{array}{l}n \\
k\end{array}\right]\left[\begin{array}{c}k \\
m\end{array}\right]$ & $\mathrm{A} 325872^{*}$ & $\sum_{k}\left[\begin{array}{l}n \\
k\end{array}\right]\left\{\begin{array}{c}k \\
m\end{array}\right\}$ & $\mathrm{A} 271703$ \\
\hline$\sum_{k}\left\{\begin{array}{l}n \\
k\end{array}\right\}\left(\begin{array}{l}k \\
m\end{array}\right)$ & $\mathrm{A} 049020$ & $\sum_{k}\left\{\begin{array}{l}n \\
k\end{array}\right\}\left[\begin{array}{c}k \\
m\end{array}\right]$ & $\mathrm{A} 129062$ & $\sum_{k}\left\{\begin{array}{l}n \\
k\end{array}\right\}\left\{\begin{array}{c}k \\
m\end{array}\right\}$ & $\mathrm{A} 130191$ \\
\hline
\end{tabular}

TABle 1. References for the sums in the On-line encyclopedia of integer sequences [7].

Closed-form expressions for two more sums are given in the next section. In the remaining cases we do not know closed forms, but the sums share many common features. Combinatorial interpretations are outlined in Section 2. Row sums of the matrix $A \cdot B$ are discussed in Section 3. Recurrences similar to Pascal's formula are proved in Section 4. Inverse relations with signed versions of the sums are given in Section 5, and in Section 6 the sums are interpreted as coefficients of change between various polynomial bases.

Our main focus are sums with $a_{n k}$ and $b_{n k}$ equal to $\left(\begin{array}{l}n \\ k\end{array}\right),\left[\begin{array}{l}n \\ k\end{array}\right]$ or $\left\{\begin{array}{l}n \\ k\end{array}\right\}$, but we include some results on sums with $L(n, k)$ as well. Our nine main sums are listed in the On-line encyclopedia of integer sequences [7] as "triangles read by rows", see Table 1 (the entry marked by * is a signed version). Many properties of the sums are reported in [7]. We repeat some properties from [7], and reveal some new properties of the sums. We mainly deal with properties that can be proved by counting arguments.

\section{COMbinatorial InTERPRETATIONS AND CLOSED FORMS}

Identity (1) follows from the usual combinatorial interpretation of Stirling numbers of the second kind. The right-hand side $\left\{\begin{array}{c}n+1 \\ m+1\end{array}\right\}$ counts partitions of an $(n+1)$-element set $S$ into $m+1$ blocks. The left-hand side is obtained by distinguishing an element of $S$ and assuming it is covered by a block containing $n-k$ other elements of $S$.

The Stirling number of the first kind $\left[\begin{array}{l}n \\ m\end{array}\right]$ is usually interpreted as the number of permutations of degree $n$ with exactly $m$ cycles. For the proof of identity (2), however, another combinatorial interpretation is more useful. Let $\Delta_{n}$ denote the board that remains after removing all tiles below and on the side diagonal of a $n \times n$ square board. Consider a new chess piece similar to a rook, but that only attacks tiles in its row. Loehr [4] calls this piece a weak rook or wrook. He goes on to 
Knežević,Krčadinac,Relić Matrix products of binomial coefficients ...

show that the number of ways of placing $k$ identical wrooks on $\Delta_{n}$ so that they don't attack each other is equal to $\left[\begin{array}{c}n \\ n-k\end{array}\right]$. The right-hand side of (2) can now be interpreted as the number of ways of placing $n-m$ non-attacking wrooks on $\Delta_{n+1}$. The left-hand side is obtained by partitioning the set of placements based on the number of wrooks not in the first column, but on the remaining $\Delta_{n}$ subboard. If $n-k$ wrooks are placed on the $\Delta_{n}$ subboard, the remaining $k-m$ wrooks must be placed in $k$ rows of the first column that are not attacked. This can be done in $\left[\begin{array}{l}n \\ k\end{array}\right]\left(\begin{array}{c}k \\ k-m\end{array}\right)=\left[\begin{array}{l}n \\ k\end{array}\right]\left(\begin{array}{l}k \\ m\end{array}\right)$ ways.

In [6], a nice combinatorial interpretation of the Lah numbers is given: $L(n, m)$ counts partitions of an $n$-element set into $m$ lists, i.e. non-empty totally ordered subsets. From this, identity (3) follows easily. Two more closed-form identities are

$$
\sum_{k=m}^{n}\left(\begin{array}{l}
n \\
k
\end{array}\right)\left(\begin{array}{l}
k \\
m
\end{array}\right)=2^{n-m}\left(\begin{array}{l}
n \\
m
\end{array}\right)
$$

and

$$
\sum_{k=m}^{n} L(n, k) L(k, m)=2^{n-m} L(n, m) .
$$

The left-hand side of (4) counts pairs $(K, M)$, where $K \supseteq M$ are subsets of a fixed $n$-element set $S$, and $|M|=m$. The right-hand side is obtained by choosing $M$ first. Identity (5) has an analogous proof using the combinatorial interpretation of Lah numbers from [6]. For two partitions $\Pi_{1}, \Pi_{2}$ of $S$ into lists, we write $\Pi_{1} \leq \Pi_{2}$ and say that $\Pi_{1}$ is a refinement of $\Pi_{2}$ if each list in $\Pi_{1}$ is a sublist of some list in $\Pi_{2}$. The left-hand side of (5) counts pairs $\left(\Pi_{1}, \Pi_{2}\right)$ with $\Pi_{1} \leq \Pi_{2}$ and $\left|\Pi_{2}\right|=m$. We first partition $S$ into $\Pi_{1}$ with $\left|\Pi_{1}\right|=k \geq m$, then partition these $k$ lists into $m$ lists containing lists of $\Pi_{1}$ as elements, and finally concatenate the list of lists into elements of $\Pi_{2}$. We get the right-hand side by partitioning $S$ into $m$ lists of $\Pi_{2}$ first and splitting them up into smaller lists of $\Pi_{1}$. This can be done in $2^{n-m}$ ways: breaks can be made in $n-m$ places, before every element except at the beginnings of the lists of $\Pi_{2}$.

Similar combinatorial interpretations can be given for the double Stirling sums $\sum_{k=m}^{n}\left\{\begin{array}{l}n \\ k\end{array}\right\}\left\{\begin{array}{c}k \\ m\end{array}\right\}$ and $\sum_{k=m}^{n}\left[\begin{array}{l}n \\ k\end{array}\right]\left[\begin{array}{l}k \\ m\end{array}\right]$. The former is the number of pairs $\left(\Pi_{1}, \Pi_{2}\right)$ of ordinary partitions of an $n$-element set into blocks (non-empty subsets), with $\Pi_{1}$ a refinement of $\Pi_{2}$ and $\left|\Pi_{2}\right|=m$. The latter is the number of pairs of permutations $\left(\pi_{1}, \pi_{2}\right)$, where $\pi_{1}$ is of degree $n, \pi_{2}$ is of degree equal to the number of cycles of $\pi_{1}$, and $\pi_{2}$ has exactly $m$ cycles. However, since we do not see an easy way to count 
Knežević,Krčadinac,Relić Matrix products of binomial coefficients ...

the pairs if $\Pi_{2}$ or $\pi_{2}$ are chosen first, this does not lead to closed-form expressions for these two sums.

Combinatorial interpretations of the remaining sums in Table 1 are as follows. The $\operatorname{sum} \sum_{k=m}^{n}\left\{\begin{array}{l}n \\ k\end{array}\right\}\left(\begin{array}{l}k \\ m\end{array}\right)$ is the number of ordinary partitions of an $n$-element set with $m$ blocks colored red, and the other blocks colored blue. The sum $\sum_{k=m}^{n}\left(\begin{array}{l}n \\ k\end{array}\right)\left[\begin{array}{l}k \\ m\end{array}\right]$ counts permutations with exactly $m$ cycles of all subsets of an $n$-element set. Finally, $\sum_{k=m}^{n}\left\{\begin{array}{c}n \\ k\end{array}\right\}\left[\begin{array}{c}k \\ m\end{array}\right]$ is the number of pairs $(\Pi, \pi)$, where $\Pi$ is a partition of an $n$-element set into blocks, and $\pi$ is a permutation with exactly $m$ cycles of degree equal to the number of blocks of $\Pi$. Again, this does not lead to closed-form expressions, but the combinatorial interpretations of the sums will be used in the following sections to prove their properties.

\section{Row SUMS}

From the combinatorial interpretation of binomial coefficients and Stirling numbers, it is clear that $\sum_{m=0}^{n}\left(\begin{array}{c}n \\ m\end{array}\right)=2^{n}, \sum_{m=0}^{n}\left[\begin{array}{c}n \\ m\end{array}\right]=n$, and $\sum_{m=0}^{n}\left\{\begin{array}{c}n \\ m\end{array}\right\}=B_{n}$. Here $B_{n}$ is the $n$-th Bell number, i.e. the total number of partitions of an $n$-element set into blocks. In this section the goal is to determine

$$
\sum_{m=0}^{n} \sum_{k=m}^{n} a_{n k} b_{k m}
$$

This is the sum of the $n$-th row of the matrix $A \cdot B$.

From identities (1) and (2), we have

$$
\sum_{m=0}^{n} \sum_{k=m}^{n}\left(\begin{array}{l}
n \\
k
\end{array}\right)\left\{\begin{array}{l}
k \\
m
\end{array}\right\}=B_{n+1} \quad \text { and } \sum_{m=0}^{n} \sum_{k=m}^{n}\left[\begin{array}{l}
n \\
k
\end{array}\right]\left(\begin{array}{l}
k \\
m
\end{array}\right)=(n+1) !
$$

Another row sum that can be written in closed form is

$$
\sum_{m=0}^{n} \sum_{k=m}^{n}\left(\begin{array}{l}
n \\
k
\end{array}\right)\left(\begin{array}{l}
k \\
m
\end{array}\right)=3^{n} .
$$

The left-hand side counts the total number of pairs $(K, M)$ of subsets of an $n$-set $S$ with $K \supseteq M$. An alternative way of counting is to decide for each element $x \in S$ whether it is in $M$, in $K \backslash M$, or in $S \backslash K$, leading to the right-hand side.

The row sum of Lah numbers

$$
\sum_{m=0}^{n} \sum_{k=m}^{n}\left[\begin{array}{l}
n \\
k
\end{array}\right]\left\{\begin{array}{l}
k \\
m
\end{array}\right\}=\sum_{m=0}^{n} L(n, m)
$$


Knežević,Krčadinac,Relić Matrix products of binomial coefficients ...

can be interpreted as the total number of partitions of an $n$-set into lists. This is the "Lah equivalent" of the Bell number $B_{n}$ and we will denote it by $L_{n}$. In [7], this is sequence number A000262.

Row sums of the remaining matrix products are given in the sequel. They have nice combinatorial interpretations and can be simplified to single sums.

$$
\sum_{m=0}^{n} \sum_{k=m}^{n}\left\{\begin{array}{l}
n \\
k
\end{array}\right\}\left[\begin{array}{l}
k \\
m
\end{array}\right]=\sum_{m=0}^{n}\left\{\begin{array}{l}
n \\
m
\end{array}\right\} m !
$$

These are the Fubini numbers, sequence A000670 in [7]. They count ordered partitions of an $n$-set, or weak orders on $n$ elements.

$$
\sum_{m=0}^{n} \sum_{k=m}^{n}\left[\begin{array}{l}
n \\
k
\end{array}\right]\left[\begin{array}{l}
k \\
m
\end{array}\right]=\sum_{m=0}^{n}\left[\begin{array}{l}
n \\
m
\end{array}\right] m !
$$

This is the number of ordered factorizations of permutations of degree $n$ into cycles, sequence A007840 in [7].

$$
\sum_{m=0}^{n} \sum_{k=m}^{n}\left\{\begin{array}{l}
n \\
k
\end{array}\right\}\left(\begin{array}{l}
k \\
m
\end{array}\right)=\sum_{m=0}^{n}\left\{\begin{array}{l}
n \\
m
\end{array}\right\} 2^{m} .
$$

The total number of partitions of an $n$-set with blocks colored red or blue. This is sequence A001861 in [7].

$$
\sum_{m=0}^{n} \sum_{k=m}^{n}\left(\begin{array}{l}
n \\
k
\end{array}\right)\left[\begin{array}{l}
k \\
m
\end{array}\right]=\sum_{m=0}^{n} \frac{n !}{m !}
$$

The total number of lists with elements from an $n$-set. Sequence number A000522 in [7].

$$
\sum_{m=0}^{n} \sum_{k=m}^{n}\left\{\begin{array}{l}
n \\
k
\end{array}\right\}\left\{\begin{array}{l}
k \\
m
\end{array}\right\}=\sum_{m=0}^{n}\left\{\begin{array}{l}
n \\
m
\end{array}\right\} B_{m} .
$$

The total number of pairs $\left(\Pi_{1}, \Pi_{2}\right)$ of partitions of an $n$-set with $\Pi_{1} \leq$ $\Pi_{2}$. In [7], this is sequence number A000258.

\section{PASCAL-Like RECURRENCES}

The binomial coefficients can be computed by Pascal's formula

$$
\left(\begin{array}{l}
n \\
m
\end{array}\right)=\left(\begin{array}{c}
n-1 \\
m-1
\end{array}\right)+\left(\begin{array}{c}
n-1 \\
m
\end{array}\right), \quad\left(\begin{array}{l}
n \\
0
\end{array}\right)=\left(\begin{array}{l}
n \\
n
\end{array}\right)=1
$$

Analogous recurrences for Stirling numbers are

$$
\left[\begin{array}{l}
n \\
m
\end{array}\right]=\left[\begin{array}{c}
n-1 \\
m-1
\end{array}\right]+(n-1)\left[\begin{array}{c}
n-1 \\
m \\
39
\end{array}\right], \quad\left[\begin{array}{l}
n \\
0
\end{array}\right]=\delta_{n 0}, \quad\left[\begin{array}{l}
n \\
n
\end{array}\right]=1,
$$


Knežević,Krčadinac,Relić Matrix products of binomial coefficients ...

$$
\left\{\begin{array}{c}
n \\
m
\end{array}\right\}=\left\{\begin{array}{c}
n-1 \\
m-1
\end{array}\right\}+m\left\{\begin{array}{c}
n-1 \\
m
\end{array}\right\},\left\{\begin{array}{c}
n \\
0
\end{array}\right\}=\delta_{n 0}, \quad\left\{\begin{array}{c}
n \\
n
\end{array}\right\}=1,
$$

and for Lah numbers

$$
L(n, m)=L(n-1, m-1)+(n+m-1) L(n-1, m)
$$

with boundary values $L(n, 0)=\delta_{n 0}$ (the Kronecker delta), $L(n, n)=1$. See [6] for proofs of the formulae by distinguishing an element of the underlying $n$-set $S$ and counting. Our sums satisfy similar recurrences that can also be established by counting arguments.

To make the formulae more readable, we denote the sum $\sum_{k=m}^{n} a_{n k} b_{k m}$ by $\left|\begin{array}{l}n \\ m\end{array}\right|$. For example, the double binomial sum $\left|\begin{array}{l}n \\ m\end{array}\right|=\sum_{k=m}^{n}\left(\begin{array}{l}n \\ k\end{array}\right)\left(\begin{array}{l}k \\ m\end{array}\right)$ can be computed from

$$
\left|\begin{array}{c}
n \\
m
\end{array}\right|=\left|\begin{array}{c}
n-1 \\
m-1
\end{array}\right|+2\left|\begin{array}{c}
n-1 \\
m
\end{array}\right|, \quad\left|\begin{array}{c}
n \\
0
\end{array}\right|=2^{n}, \quad\left|\begin{array}{l}
n \\
n
\end{array}\right|=1 .
$$

This sum also satisfies the absorption identity $\left|\begin{array}{l}n \\ m\end{array}\right|=\frac{n}{m}\left|\begin{array}{l}n-1 \\ m-1\end{array}\right|$, just like the binomial coefficients.

By (1), the sum $\left|\begin{array}{l}n \\ m\end{array}\right|=\sum_{k=m}^{n}\left(\begin{array}{l}n \\ k\end{array}\right)\left\{\begin{array}{c}k \\ m\end{array}\right\}$ are shifted Stirling numbers of the second kind. Therefore,

$$
\left|\begin{array}{c}
n \\
m
\end{array}\right|=\left|\begin{array}{c}
n-1 \\
m-1
\end{array}\right|+(m+1)\left|\begin{array}{c}
n-1 \\
m
\end{array}\right|, \quad\left|\begin{array}{l}
n \\
0
\end{array}\right|=\left|\begin{array}{l}
n \\
n
\end{array}\right|=1 .
$$

Similarly, by (2), the sum $\left|\begin{array}{l}n \\ m\end{array}\right|=\sum_{k=m}^{n}\left[\begin{array}{l}n \\ k\end{array}\right]\left(\begin{array}{l}k \\ m\end{array}\right)$ satisfies

$$
\left|\begin{array}{c}
n \\
m
\end{array}\right|=\left|\begin{array}{c}
n-1 \\
m-1
\end{array}\right|+n\left|\begin{array}{c}
n-1 \\
m
\end{array}\right|, \quad\left|\begin{array}{c}
n \\
0
\end{array}\right|=n !, \quad\left|\begin{array}{l}
n \\
n
\end{array}\right|=1 .
$$

The sum $\left|\begin{array}{l}n \\ m\end{array}\right|=\sum_{k=m}^{n}\left(\begin{array}{l}n \\ k\end{array}\right) L(k, m)$ is sequence A271705 in [7], where the following recurrence is given:

$$
\left|\begin{array}{c}
n \\
m
\end{array}\right|=\frac{n}{m}\left|\begin{array}{c}
n-1 \\
m-1
\end{array}\right|+n\left|\begin{array}{c}
n-1 \\
m
\end{array}\right|, \quad\left|\begin{array}{c}
n \\
0
\end{array}\right|=\left|\begin{array}{c}
n \\
n
\end{array}\right|=1 .
$$

The sum $\left|\begin{array}{l}n \\ m\end{array}\right|=\sum_{k=m}^{n} L(n, k)\left(\begin{array}{l}k \\ m\end{array}\right)$ is sequence A059110. It satisfies the same recurrence, but with different boundary values $\left|\begin{array}{l}n \\ 0\end{array}\right|=L_{n}$.

The double Lah sum $\left|\begin{array}{l}n \\ m\end{array}\right|=\sum_{k=m}^{n} L(n, k) L(k, m)$ satisfies

$$
\left|\begin{array}{c}
n \\
m
\end{array}\right|=\frac{n}{m}\left|\begin{array}{c}
n-1 \\
m-1
\end{array}\right|+2 n\left|\begin{array}{c}
n-1 \\
m
\end{array}\right|, \quad\left|\begin{array}{c}
n \\
0
\end{array}\right|=\delta_{n 0}, \quad\left|\begin{array}{c}
n \\
n
\end{array}\right|=1 .
$$

This sum also satisfies the absorption-like identity $\left|\begin{array}{c}n \\ m\end{array}\right|=\frac{n-m+1}{2 m(m-1)}\left|\begin{array}{c}n \\ m-1\end{array}\right|$, while the Lah numbers satisfy $L(n, m)=\frac{n-m+1}{m(m-1)} L(n, m-1)$. 
Knežević,Krčadinac,Relić Matrix products of binomial coefficients ...

Now let $\left|\begin{array}{l}n \\ m\end{array}\right|=\sum_{k=m}^{n}\left(\begin{array}{l}n \\ k\end{array}\right)\left[\begin{array}{l}k \\ m\end{array}\right]$. This sum cannot be computed from the two values $\left|\begin{array}{c}n-1 \\ m-1\end{array}\right|$ and $\left|\begin{array}{c}n-1 \\ m\end{array}\right|$ alone, but we can give a Pascal-like recurrence involving more previous values:

$$
\left|\begin{array}{c}
n \\
m
\end{array}\right|=\sum_{k=0}^{n-m}(n-1)^{\underline{k}}\left|\begin{array}{c}
n-1-k \\
m-1
\end{array}\right|+\left|\begin{array}{c}
n-1 \\
m
\end{array}\right|, \quad\left|\begin{array}{l}
n \\
0
\end{array}\right|=\left|\begin{array}{l}
n \\
n
\end{array}\right|=1 .
$$

Here $(n-1)^{\underline{k}}=(n-1) \cdot(n-2) \cdots(n-k)$ is the falling factorial. For the proof, recall that $\left|\begin{array}{l}n \\ m\end{array}\right|$ counts permutations with $m$ cycles of subsets $T \subseteq S$, where $S$ is a set of $n$ elements. Fix an element $x \in S$ and divide the permutations according to whether they contain $x$ or do not contain $x$. In the latter case there are clearly $\left|\begin{array}{c}n-1 \\ m\end{array}\right|$ permutations. In the former case, assume $x$ is in a cycle with $k$ other elements of $S$. This cycle can be chosen in $(n-1)^{\underline{k}}$ ways, and $m-1$ more cycles have to be chosen from the remaining $n-1-k$ elements. Thus, there are $\sum_{k=0}^{n-m}(n-1)^{\underline{k}}\left|\begin{array}{c}n-1-k \\ m-1\end{array}\right|$ permutations containing $x$.

The sum $\left|\begin{array}{l}n \\ m\end{array}\right|=\sum_{k=m}^{n}\left\{\begin{array}{l}n \\ k\end{array}\right\}\left(\begin{array}{l}k \\ m\end{array}\right)$ counts partitions of $S$ with $m$ blocks colored red, and the other blocks colored blue. Again, fix an element $x \in S$. If $x$ is in a red block alone, there are $\left|\begin{array}{c}n-1 \\ m-1\end{array}\right|$ partitions. If $x$ is in a red block with some other elements of $S$, there are $m\left|\begin{array}{c}n-1 \\ m\end{array}\right|$ partitions. Finally, if $x$ is in a blue block with $k$ other elements of $S$, there are $\left(\begin{array}{c}n-1 \\ k\end{array}\right)\left|\begin{array}{c}n-1-k \\ m\end{array}\right|$ such partitions. Therefore, the following recursion holds:

$$
\left|\begin{array}{c}
n \\
m
\end{array}\right|=\left|\begin{array}{c}
n-1 \\
m-1
\end{array}\right|+m\left|\begin{array}{c}
n-1 \\
m
\end{array}\right|+\sum_{k=0}^{n-m-1}\left(\begin{array}{c}
n-1 \\
k
\end{array}\right)\left|\begin{array}{c}
n-1-k \\
m
\end{array}\right| .
$$

The boundary values are $\left|\begin{array}{l}n \\ 0\end{array}\right|=B_{n}$ and $\left|\begin{array}{l}n \\ n\end{array}\right|=1$.

The sum $\left|\begin{array}{l}n \\ m\end{array}\right|=\sum_{k=m}^{n}\left\{\begin{array}{l}n \\ k\end{array}\right\}\left\{\begin{array}{c}k \\ m\end{array}\right\}$ counts pairs $\left(\Pi_{1}, \Pi_{2}\right)$ of partitions of $S$, where $\Pi_{2}$ has $m$ blocks and $\Pi_{1}$ is a refinement of $\Pi_{2}$. Now let the fixed element $x \in S$ be contained in a block of $\Pi_{2}$ of size $k$. We can choose this block in $\left(\begin{array}{c}n-1 \\ k-1\end{array}\right)$ ways and partition it into blocks of $\Pi_{1}$ in $B_{k}$ ways. The remaining blocks of $\Pi_{2}$ and $\Pi_{1}$ can be chosen in $\left|\begin{array}{c}n-k \\ m-1\end{array}\right|$ ways. Therefore,

$$
\left|\begin{array}{l}
n \\
m
\end{array}\right|=\sum_{k=1}^{n-m+1}\left(\begin{array}{l}
n-1 \\
k-1
\end{array}\right) B_{k}\left|\begin{array}{c}
n-k \\
m-1
\end{array}\right|, \quad\left|\begin{array}{l}
n \\
0
\end{array}\right|=\delta_{n 0}, \quad\left|\begin{array}{l}
n \\
n
\end{array}\right|=1 .
$$

For the sum $\left|\begin{array}{l}n \\ m\end{array}\right|=\sum_{k=m}^{n}\left[\begin{array}{l}n \\ k\end{array}\right]\left[\begin{array}{l}k \\ m\end{array}\right]$ a similar argument leads to the recurrence

$\left|\begin{array}{l}n \\ m\end{array}\right|=\sum_{k=1}^{n-m+1}\left(\begin{array}{l}n-1 \\ k-1\end{array}\right) \sum_{i=1}^{k}\left[\begin{array}{l}k \\ i\end{array}\right](i-1) !\left|\begin{array}{c}n-k \\ m-1\end{array}\right|,\left|\begin{array}{l}n \\ 0\end{array}\right|=\delta_{n 0}, \quad\left|\begin{array}{l}n \\ n\end{array}\right|=1$, 
Knežević,Krčadinac,Relić Matrix products of binomial coefficients ... and for the sum $\left|\begin{array}{l}n \\ m\end{array}\right|=\sum_{k=m}^{n}\left\{\begin{array}{l}n \\ k\end{array}\right\}\left[\begin{array}{c}k \\ m\end{array}\right]$ to the recurrence $\left|\begin{array}{l}n \\ m\end{array}\right|=\sum_{k=1}^{n-m+1}\left(\begin{array}{l}n-1 \\ k-1\end{array}\right) \sum_{i=1}^{k}\left\{\begin{array}{l}k \\ i\end{array}\right\}(i-1) !\left|\begin{array}{c}n-k \\ m-1\end{array}\right|,\left|\begin{array}{l}n \\ 0\end{array}\right|=\delta_{n 0}, \quad\left|\begin{array}{l}n \\ n\end{array}\right|=1$.

However, these increasingly complex recurrences become less useful as the coefficients are more difficult to evaluate than the sum $\left|\begin{array}{l}n \\ m\end{array}\right|$ directly.

\section{INVERSES}

We denote signed versions of the Stirling numbers and their relatives by an exponent ${ }^{\sigma}$, e.g. $\left[\begin{array}{c}n \\ m\end{array}\right]^{\sigma}=(-1)^{n-m}\left[\begin{array}{c}n \\ m\end{array}\right]$. For the matrix $A=\left[a_{n m}\right]$, we denote $A^{\sigma}=\left[a_{n m}^{\sigma}\right]=\left[(-1)^{n-m} a_{n m}\right]$. To avoid excessive bracketing, we write $\left[\begin{array}{c}n \\ m\end{array}\right]^{-1}$ for the inverse matrix $A^{-1}$.

It is well-known that $\left(\begin{array}{c}n \\ m\end{array}\right)^{-1}=\left(\begin{array}{c}n \\ m\end{array}\right)^{\sigma},\left[\begin{array}{c}n \\ m\end{array}\right]^{-1}=\left\{\begin{array}{c}n \\ m\end{array}\right\}^{\sigma}$, and $\left\{\begin{array}{c}n \\ m\end{array}\right\}^{-1}=$ $\left[\begin{array}{c}n \\ m\end{array}\right]^{\sigma}$. From this and the properties $(A \cdot B)^{-1}=B^{-1} \cdot A^{-1}$ and $(A \cdot B)^{\sigma}=$ $A^{\sigma} \cdot B^{\sigma}$, we can determine inverses of our sums. For example, let $a_{m n}=\left[\begin{array}{c}n \\ m\end{array}\right]$ and $b_{m n}=\left\{\begin{array}{c}n \\ m\end{array}\right\}$. Then $A \cdot B$ are the Lah numbers $L(n, m)$ and we have

$$
L(n, m)^{-1}=(A \cdot B)^{-1}=B^{-1} \cdot A^{-1}=A^{\sigma} \cdot B^{\sigma}=(A \cdot B)^{\sigma}=L(n, m)^{\sigma} .
$$

Similarly it follows

$$
\begin{aligned}
& \left(\sum_{k=m}^{n}\left(\begin{array}{l}
n \\
k
\end{array}\right)\left\{\begin{array}{c}
k \\
m
\end{array}\right\}\right)^{-1}=\left(\sum_{k=m}^{n}\left[\begin{array}{l}
n \\
k
\end{array}\right]\left(\begin{array}{c}
k \\
m
\end{array}\right)\right)^{\sigma}, \\
& \left(\sum_{k=m}^{n}\left\{\begin{array}{l}
n \\
k
\end{array}\right\}\left(\begin{array}{c}
k \\
m
\end{array}\right)\right)^{-1}=\left(\sum_{k=m}^{n}\left(\begin{array}{l}
n \\
k
\end{array}\right)\left[\begin{array}{c}
k \\
m
\end{array}\right]\right)^{\sigma}, \\
& \left(\sum_{k=m}^{n}\left\{\begin{array}{l}
n \\
k
\end{array}\right\}\left\{\begin{array}{c}
k \\
m
\end{array}\right\}\right)^{-1}=\left(\sum_{k=m}^{n}\left[\begin{array}{l}
n \\
k
\end{array}\right]\left[\begin{array}{c}
k \\
m
\end{array}\right]\right)^{\sigma},
\end{aligned}
$$

and

$$
\left(\sum_{k=m}^{n}\left\{\begin{array}{l}
n \\
k
\end{array}\right\}\left[\begin{array}{l}
k \\
m
\end{array}\right]\right)^{-1}=\left(\sum_{k=m}^{n}\left\{\begin{array}{l}
n \\
k
\end{array}\right\}\left[\begin{array}{l}
k \\
m
\end{array}\right]\right)^{\sigma} .
$$

The exponents ${ }^{-1}$ and ${ }^{\sigma}$ can be exchanged in the formulae above. 
Knežević,Krčadinac,Relić Matrix products of binomial coefficients ...

\section{Polynomial Bases}

We denote the falling factorials by $x^{\underline{n}}=x(x-1) \cdots(x-n+1)$ and the rising factorials by $x^{\bar{n}}=x(x+1) \cdots(x+n-1)$, following [1]. It is known that Stirling numbers of the second kind are coefficients of change from the standard polynomial basis of powers $\left(x^{n} \mid n \in \mathbb{N}_{0}\right)$ to the basis of falling factorials $\left(x^{\underline{m}} \mid m \in \mathbb{N}_{0}\right)$ :

$$
x^{n}=\sum_{m=0}^{n}\left\{\begin{array}{c}
n \\
m
\end{array}\right\} x^{\underline{m}} .
$$

Stirling numbers of the first kind are coefficients of change from $\left(x^{\bar{n}}\right)$ to $\left(x^{m}\right)$ :

$$
x^{\bar{n}}=\sum_{m=0}^{n}\left[\begin{array}{l}
n \\
m
\end{array}\right] x^{m} .
$$

From (6) and (7) it follows that coefficients of change from $\left(x^{\bar{n}}\right)$ to $\left(x^{\underline{m}}\right)$ are the Lah numbers $L(n, m)=\sum_{k=m}^{n}\left[\begin{array}{l}n \\ k\end{array}\right]\left\{\begin{array}{c}k \\ m\end{array}\right\}$ :

$$
x^{\bar{n}}=\sum_{m=0}^{n} L(n, m) x^{\underline{m}} \text {. }
$$

Ivo Lah's original definition of his numbers [2, 3] was a signed version of this relation. We concentrate on changes between polynomial bases with non-negative coefficients. The opposite changes have inverse coefficients, with alternating signs as shown in the previous section.

From the binomial theorem $(1+x)^{n}=\sum_{m=0}^{n}\left(\begin{array}{c}n \\ m\end{array}\right) x^{m}$ and (6) we see that the sums $\sum_{k=m}^{n}\left(\begin{array}{l}n \\ k\end{array}\right)\left\{\begin{array}{l}k \\ m\end{array}\right\}$ can be interpreted as coefficients of change from the polynomial basis $\left((1+x)^{n}\right)$ to the basis of falling factorials $\left(x^{\underline{m}}\right)$ :

$$
(1+x)^{n}=\sum_{m=0}^{n}\left(\sum_{k=m}^{n}\left(\begin{array}{l}
n \\
k
\end{array}\right)\left\{\begin{array}{l}
k \\
m
\end{array}\right\}\right) x^{\underline{m}} .
$$

Similar interpretations can be given to other sums from Table 1. The double binomial sums $\sum_{k=m}^{n}\left(\begin{array}{l}n \\ k\end{array}\right)\left(\begin{array}{l}k \\ m\end{array}\right)$ are coefficients of change from the basis $\left((2+x)^{n}\right)$ to the standard basis $\left(x^{m}\right)$. The sums $\sum_{k=m}^{n}\left(\begin{array}{l}n \\ k\end{array}\right)\left[\begin{array}{l}k \\ m\end{array}\right]$ are coefficients of change from the basis $\left(\sum_{k=0}^{n}\left(\begin{array}{l}n \\ k\end{array}\right) x^{\bar{k}}\right)$ to the standard basis. The former basis contains a special case of Charlier polynomials [7, a family of orthogonal polynomials that can be written in terms of the generalized hypergeometric function (see [5]). Double Stirling sums of the second kind $\sum_{k=m}^{n}\left\{\begin{array}{l}n \\ k\end{array}\right\}\left\{\begin{array}{c}k \\ m\end{array}\right\}$ are coefficients of change from the basis of Bell polynomials $\left(B_{n}(x)\right), B_{n}(x)=\sum_{k=0}^{n}\left\{\begin{array}{c}n \\ k\end{array}\right\} x^{k}$ to the basis of falling factorials $\left(x^{\underline{m}}\right)$. 
Knežević,Krčadinac,Relić Matrix products of binomial coefficients ...

The two families of sums $\sum_{k=m}^{n}\left\{\begin{array}{l}n \\ k\end{array}\right\}\left[\begin{array}{l}k \\ m\end{array}\right]$ and $\sum_{k=m}^{n}\left[\begin{array}{l}n \\ k\end{array}\right]\left[\begin{array}{c}k \\ m\end{array}\right]$ can be seen as coefficients of the polynomials $\sum_{k=0}^{n}\left\{\begin{array}{l}n \\ k\end{array}\right\} x^{\bar{k}}$ and $\sum_{k=0}^{n}\left[\begin{array}{l}n \\ k\end{array}\right] x^{\bar{k}}$, i.e. coefficients of change to the standard basis $\left(x^{m}\right)$. Similarly, the two families $\sum_{k=m}^{n}\left\{\begin{array}{l}n \\ k\end{array}\right\}\left(\begin{array}{l}k \\ m\end{array}\right)$ and $\sum_{k=m}^{n}\left[\begin{array}{l}n \\ k\end{array}\right]\left(\begin{array}{l}k \\ m\end{array}\right)$ are coefficients of Bell polynomials $B_{n}(1+x)$ and polynomials $\sum_{k=0}^{n}\left[\begin{array}{l}n \\ k\end{array}\right](1+x)^{k}$, respectively.

\section{ACKNOWLEDGMENT}

The second author has been supported by the Croatian Science Foundation under the project 6732 .

\section{REFERENCES}

[1] R. L. Graham, D. E. Knuth, O. Patashnik, Concrete Mathematics, 2nd edition, Addison-Wesley Publishing Company, 1994.

[2] I. Lah, A new kind of numbers and its application in the actuarial mathematics, Boletim do Instituto dos Actuários Portugueses 9 (1954), 7-15.

[3] I. Lah, Eine neue Art von Zahlen, ihre Eigenschaften und Anwendung in der mathematischen Statistik, Mitteilungsbl. Math. Statist. 7 (1955), 203-212.

[4] N. A. Loehr, Bijective Combinatorics, CRC Press, 2011.

[5] N. Özmen, E. Erkuş-Duman, On the Poisson-Charlier polynomials, Serdica Math. J. 41 (2015), no. 4, 457-470.

[6] M. Petkovšek, T. Pisanski, Combinatorial interpretation of unsigned Stirling and Lah numbers, The Pi Mu Epsilon Journal 12 (2007), No. 7, 417-424.

[7] N. J. A. Sloane (editor), The On-Line Encyclopedia of Integer Sequences, https://oeis.org (accessed December, 2020).

Email address: marin.k59@gmail.com

Email address: vedran.krcadinac@math.hr

Department of Mathematics, Faculty of Science, University of ZaGReb, BijeničKa 30, HR-10000 Zagreb, Croatia

Email address: lucijarelic7@gmail.com 